\title{
La atracción en la elección de pareja
}

\author{
Hellen PUMA SIMBRÓN*
}

E.A.P. Psicología, Facultad de Ciencias de la Salud, Universidad Peruana Unión, Alt. Km 19; Carretera Central, Lurigancho, Lima

\begin{abstract}
RESUMEN
El objetivo de la investigación es analizar el papel que cumple la atracción en la elección de la pareja y los factores que están involucrados en el contexto social. Se trata de un artículo de revisión teórica. Se presenta al atractivo físico como un elemento importante a la hora de elegir una pareja, ya que impacta de manera positiva en las personas y esto abre mayores oportunidades; sin embargo, se debe tener en cuenta que el concepto de belleza depende del contexto social y cultural. Se concluye que la atracción es el punto inicial al momento de elegir una pareja, esto involucra el atractivo físico y otros factores similares; sin embargo, debe considerarse también el contexto social (cultura, costumbres, valores, principios) que rodea a cada individuo, ya que eso influye en su concepto de lo que es bello.
\end{abstract}

Palabras clave: Atracción, atractivo físico, belleza, contexto social.

\begin{abstract}
The objective of the research is to analyze the role of attraction in mate choice and the factors that are involved in the social context. This is a theoretical review article. It comes to physical attractiveness as an important element when choosing a partner, as it impacts positively on people and this opens up more opportunities, but it should be noted that the concept of beauty depends on the social context and cultural. We conclude that the attraction is the starting point when choosing a partner, this involves physical attractiveness and other similar factors, but must also consider the social context (culture, customs, values, principles) that surrounds each individual, as this affects their concept of what is beautiful.
\end{abstract}

Keywords: Attraction, attractiveness, beauty, social context.

*Correspondencia: helenapuma7@gmail.com 


\section{INTRODUCCIÓN}

A lo largo de la historia, la atracción ha jugado un papel fundamental en las relaciones interpersonales, tanto en la amistad como en la pareja; sin embargo, su estudio se ha incrementado en los últimos años. La pregunta principal que muchos han querido responder es ¿qué le atrae a una persona de otra?, ¿ ¿es la atracción física o existen otros factores que intervienen?

Muchas investigaciones actuales han referido que todo depende de las pautas culturales de cada persona, otros que depende de la atracción, un ejemplo de ello es el estudio realizado por Barriera, Soler, Jarrosay y Montoya (1999), donde se observó que el motivo principal para elegir a una pareja en los adolescentes es la atracción física. Asimismo, Díaz, Pandolfi y Perfetti (2001), determinaron la importancia del atractivo físico en las relaciones interpersonales, siendo éste un constructo social determinado culturalmente y que varía según patrones de estética raciales y tendencias de la moda. Por otro lado, en la investigación hecha por Valdez, González y Sánchez (2005), se encontró que las características que los jóvenes toman en cuenta al momento de elegir pareja son similares en el hombre que en la mujer, ambos tienen la finalidad común de satisfacer sus necesidades propias de afiliación. Respecto al ámbito cultural y la atracción, Valdez, González, Arce, González, Ison y Morelato (2008), en base a sus resultados, concluyeron que para algunos la elección de pareja se guía por los tradicionales roles de género, mientras que para otros se ve influenciada por un pensamiento posmoderno.

Como se puede ver, las propuestas referidas al tema mencionado son diversas, por este motivo se analizará el papel que cumple la atracción en la elección de pareja y los factores que están involucrados en el contexto social.

\section{Definiciones}

Según el Diccionario Manual de la Lengua Española (2007), atracción significa la acción de atraer (acercar), es decir la fuerza que atrae el interés o inclinación hacia alguien o algo.

Para entender las relaciones de pareja, es necesario comprender qué los atrae, en cuanto a esto se debe considerar que para los seres humanos, más que para ninguna otra especie, son importantes las necesidades de apareamiento, compatibilidad física y biológica (Ostrowsky, 2005 y Schopenauer, 2004), afecto, apego, cuidado, cariño, interdependencia, compañía y amor, que son necesidades genéticamente fundamentales y determinantes para la supervivencia de la especie
(Buss, 2006; Díaz y Sánchez, 2002).

De esta manera Buss (2006), dice que la atracción es uno de los factores que intervienen en la elección de la pareja, este proceso se compone de cuatro diferentes etapas secuenciadas: la atracción y elección, el cortejo, la conservación y la posible ruptura con la pareja, esta búsqueda y elección tiene como base biológica a la reproducción. Sin embrago, al momento de elegir una pareja no solo se pone en juego la atracción sino también las diferentes circunstancias, normas, reglas o guías de vida social que imponen las culturas y, por supuesto, las necesidades y bases psicológicas que cada persona posee.

En términos generales, la atracción hacia un miembro del sexo opuesto (con base en las características específicas de cada especie y de cada individuo) genera el emparejamiento (elección de pareja) de manera natural (Attenborough, 2005 y Schopenhauer, 2004).

A partir de esta perspectiva, tanto Buss (2006), como Díaz y Sánchez (2002), y Valdez, Díaz y Pérez (2006), han encontrado que el atractivo físico es un factor importante para los hombres, en cuanto a su elección de pareja, ya que buscan una mujer que les sea atractiva por su físico, que muestre una salud favorable y que posea forma de "reloj de arena" (pechos abundantes para amamantar adecuadamente a las crías y caderas amplias que aseguren la protección del feto ante cualquier amenaza o incidente desfavorable que pudiera llegar a sufrir). En contraste, la atracción en las mujeres está relacionada con el hecho de elegir hombres que sean óptimos proveedores, que tengan habilidades suficientes para conseguir recursos, un buen estatus socioeconómico y, sobre todo, que estén dispuestos a invertir sus recursos en ellas y en las hijos que lleguen a procrear.

\section{Reseña histórica}

En el principio, cuando Dios creó a los seres humanos los hizo perfectos física y psicológicamente "Y creó Dios al hombre a su imagen, a imagen de Dios lo creó; varón y hembra los creó. [ ...] Y vio Dios todo lo que había hecho, y he aquí que era bueno en gran manera. Y fue la tarde y la mañana el día sexto (Génesis 1: 27, 31)". La finalidad de crear un hombre y una mujer fue para que se unieran y complementaran formando una pareja, un matrimonio "Por tanto, dejará el hombre a su padre y a su madre, y se unirá a su mujer, y serán una sola carne (Génesis 2: 24)”. La atracción por el sexo opuesto junto con la elección de la pareja ha sido establecida desde nuestra creación como un rasgo característico del ser humano.

La pareja a lo largo de la historia ha sido establecida 
como el núcleo de la sociedad. Pero antiguamente no existía una elección voluntaria de la pareja, pues era vista como un contrato de negocio en el que los más beneficiados eran los padres o mejor dicho las familias a la que representaban. Es a inicios del siglo XIX cuando las parejas se empiezan a formar en base a su propia elección, pero esto dio paso a otro dilema, ¿qué hace que las personas se unan como pareja? (Antaki, 1999).

En el siglo XIX, Schopenhauer decía que la elección de pareja se relacionaba exclusivamente con la reproducción, por este motivo la elección dependía del atractivo físico de la persona a la que se elegía, lo cual involucraba la simetría del cuerpo, los rasgos faciales, y las hormonas que despedía el cuerpo, pues esto podría producir la excitación sexual de la pareja (Ostrowsky, 2005).

Por otro lado, la teoría de la complementariedad decía que una persona elegía a otra no por la similitud con ella sino por la complementariedad, es decir que uno se sentiría atraído a otro porque esa persona tendría aquello que a uno le faltaba (Winch, 1958).

Otros factores importantes son mencionados por Padilla y Díaz (2002), quienes proponían que la atracción en la elección de pareja no solo estaba dada por la similitud o la complementariedad, sino que había otros factores que también influían, como el factor socioeconómico, político, religioso y psicológico de la persona que elegía.

Asímismo, Álvarez (1996), refirió que al comienzo la atracción física es de suma importancia en una relación de pareja; sin embargo, después continúa una atracción afectiva e intelectual que fortalece la relación. Es en esta última etapa en donde la religión, el nivel económico, educación, raza y edad, juegan un papel relevante; en otras palabras la atracción conformaría un proceso que involucraría factores personales, psicológicos, sociales y emocionales (Rice, 1997).

Actualmente, debido a los cambios ideológicos y culturales con tendencia a la igualdad de sexos, se ha producido un cambio respecto al concepto y características que favorecen la atracción en la elección de pareja tanto de hombres como mujeres, pues ambos desean cosas diferentes en una relación. Martínez y Sternberg explican que:

Por término medio, las mujeres desean un varón con capacidad económica, estatus social, edad, ambición, laboriosidad, formalidad, estabilidad, inteligencia, compatibilidad, estatura, fuerza, buena salud, amor y compromiso, en comparación con los hombres, que prefieren una mujer joven, físicamente hermosa, con un cuerpo atractivo, casta y fiel (Martínez y Sternberg citados por Valdés, Gonzales y Sánchez, 2005, p. 5).

\section{Características de la atracción en la elección de pareja}

\section{Atractivo físico y contexto social}

Cuando se habla de atracción se hace referencia a las relaciones interpersonales, ya que se trata de ver qué características o rasgos son los que atraen a las personas. Uno de los que se considera es el atractivo físico, ya que según los sociólogos es lo primero que impacta en los demás. Días, Pandolfi y Perfetti (2001), refieren también que la belleza depende de los ojos con los que se la vea, es decir del concepto que se tenga de lo que es bello, lo cual obedece a las pautas culturales que una persona tenga; un ejemplo de ello son los atributos físicos, pues para algunos podrían tener relación con la capacidad de reproducción del sexo opuesto, de esta manera se dice que una mujer es atractiva solo si tiene características relacionadas con la fertilidad y la juventud, y los hombres rasgos de dominio, fuerza y un buen estatus social.

\section{El tono de voz}

Otro de los elementos o factores del atractivo físico es el tono de voz, para algunos una voz sumisa es mucho más atractiva que la dominante. Para otros lo atractivo tiene que ver con la imagen, en relación a esto:

Las investigaciones demuestran que las personas que están bronceadas son consideradas más físicamente atractivas, populares y sexy que aquellas que no están en esa condición, existiendo una preferencia por un bronceado mediano sobre uno débil, con una clara menor preferencia para un bronceado oscuro y una ausencia total de éste (Broadstock citado por Díaz et al., 2001, p. 5).

\section{El maquillaje y la buena postura}

Existen otros factores que influyen en la atracción hacia los demás, por ejemplo, la calvicie es considerada poco atrayente, poco deseable, puesto que los hombres con calvicie son vistos como más viejos, en otras palabras se relaciona a la calvicie con la edad avanzada. Por otro lado, el uso de maquillaje y la buena postura son vistos como algo atractivo para algunas personas: Se diseñó una investigación para determinar en qué medida los sujetos pueden influenciar sus niveles de atractivo físico, centrándose específicamente en el uso de maquillaje y el uso de la postura. Se vio que la estructura física es tan importante como la estructura facial al momento de establecer juicios sobre niveles de atractivo físico, se demostró, por lo tanto, que hay factores comportamentales que influyen sobre los juicios de belleza y que son casi tan importantes como los factores estructurales (Díaz et al., 2001, p. 5, 6). 


\section{El atractivo físico y las relaciones interpersonales}

Por otro lado, se comenta que el atractivo físico es una fuente de influencia en las relaciones sociales, lo cual puede ser tomado como positivo a la hora de elegir un líder, Cherulnik opina lo siguiente:

En cuanto al liderazgo una persona será considerada más exitosa, con mayores habilidades sociales, ambicioso y competente cuando es físicamente atractivo; por lo tanto, el atractivo físico cumpliría un rol importante en la elección del líder; lo que se traduce en una profecía autocumplida, lo cual lleva al líder, que en un principio fue elegido por su atractivo físico a ser un líder efectivo (Díaz et al., 2001, p. 3).

Se puede notar que el atractivo físico es considerado como un elemento importante a la hora de elegir con quién relacionarse, esto no significa que los que no lo posean sean personas solitarias, sino que el atractivo físico impacta de manera positiva en las personas y esto abre mayores oportunidades a la hora de elegir a una pareja, un amigo o un líder de grupo. Sin embargo, se debe tener en cuenta que el concepto de belleza no es estático, depende de los parámetros del sujeto, de su contexto social y cultural, es por eso que muchas personas tienen una percepción distinta de belleza a comparación de otras, debido a que el concepto de atractivo físico varía de acuerdo a cada persona.

\section{Importancia del atractivo físico y sus componentes}

De acuerdo a Ruiz (1999), la apariencia física es muy importante para el ser humano, es su carta de presentación, la primera impresión; sin embargo, a lo largo de la vida la apariencia va cambiando, y el ser humano debe aprender a aceptar esos cambios y adaptarse para que no lo afecte emocionalmente. Por esta razón, el atractivo físico juega un papel relevante en la vida social de cada uno, especialmente la belleza facial.

\section{El atractivo facial}

El atractivo facial es uno de los componentes del atractivo físico en general; aunque este componente depende básicamente de los valores culturales de cada persona, se puede decir que el atractivo facial está relacionado con los “ojos grandes, nariz pequeña, barbilla pequeña, pómulos prominentes, mejillas estrechas, cejas altas, pupilas dilatadas y amplia sonrisa”, éstas serían las características de una cara bella, según Etcoff (1994).

\section{El atractivo físico como punto inicial para la elección de pareja}

Si bien la apariencia física es un punto esencial en la atracción hacia el sexo opuesto, la percepción de lo que es bello no siempre es la misma, todo dependerá de los valores, costumbres, cultura, etc. Sin embargo, la elección de pareja es un hecho, y la atracción es el punto inicial por el que una persona se acerca a otra. Existe un mandato divino que establece la aceptación mutua entre el hombre y la mujer, "Por tanto, acéptense mutuamente, así como Cristo los aceptó a ustedes para gloria de Dios (Romanos 15: 7)”; esta aceptación va más allá de la atracción, ya que tiene que ver con el amor. En otras palabras se diría que la atracción junto con el atractivo físico son el punto inicial, el primer paso hacia la elección de una pareja, pero no el único, existen otros pasos importantes también, como la forma de pensar, el estilo de vida, los objetivos de vida, etc.

\section{Investigaciones sobre atracción interpersonal}

Valdez, González y Sánchez (2005), en su investigación titulada "Elección de pareja en universitarios mexicanos”, estudiaron las características que los universitarios toman en cuenta al momento de elegir una pareja real e ideal. Se trabajó con una muestra de 100 sujetos mediante la técnica de redes semánticas naturales. Los resultados muestran que para elegir pareja existen más similitudes que diferencias entre hombres y mujeres. Se descubrió que las mujeres toman en cuenta las emociones, el humor, la intimidad, la personalidad y los valores, mientras que los hombres las cualidades físicas, la intimidad y la personalidad. Los resultados respecto a la pareja fueron similares, ya que las mujeres definen su relación en base a las emociones, valores, personalidad y cualidades físicas, y los hombres en función de la intimidad, el humor, la personalidad y las cualidades físicas. Es decir, ambos tienen el objetivo de satisfacer sus necesidades propias de afiliación.

Valdez et al (2008), en su trabajo titulado "La elección de pareja real e ideal en dos culturas: México y Argentina. Un análisis por sexo”, compararon a mexicanos y argentinos respecto de las características de elección de pareja. Se trabajó con 200 universitarios repartidos equitativamente por nacionalidad y por sexo en las ciudades de Toluca (México) y Mendoza (Argentina), empleándose para ello la técnica de redes semánticas. Los resultados muestran que, en general, en México las personas eligen a su pareja en base a los roles tradicionales de género de esa cultura, en cambio en Argentina se ve influida por un pensamiento posmoderno.

Barriera, Soler, Jarrosay y Montoya (1999), realizaron una investigación con el propósito de determinar los criterios para elegir pareja. Trabajaron con un grupo del noveno grado de la secundaria básica "Francisco Maceo Osorio" (Santiago de Cuba), durante el curso escolar 1996 - 1997. Se seleccionó una muestra alea- 
toria a la que se aplicó una encuesta; en el grupo, las edades más frecuentes oscilaron entre 12 y 14 años, con predominio del sexo femenino. Entre los motivos para la elección figuraron, en primer término, el criterio de otros compañeros y la atracción física; la mayoría cambiaba frecuentemente de pareja y un alto porcentaje mantenía relaciones sexuales, mientras que la mayor información sobre educación sexual la recibieron de los amigos.

Díaz, Pandolfi y Perfetti (2001), realizaron un estudio bibliográfico con el fin de determinar la importancia del atractivo físico en las relaciones interpersonales y como ésta afecta el autoimagen. Se concluyó que el atractivo físico es un constructo social determinado culturalmente y que varía según patrones de estética raciales y tendencias de la moda, que influirán sobre el establecimiento de relaciones sociales, sobre las posiciones de liderazgo, el establecimiento del autoimagen y juicios sobre la propia imagen corporal, sobre conductas que se presentan y, por último, se vio que hay diferencias de género en cuanto al tema.

Ruiz (1999), en su trabajo "Evaluación del atractivo físico de modelos audiovisuales: procesos de identificación en niños y adolescentes” analizó cómo los medios de comunicación audiovisuales ejercen influencia en el conocimiento social de niños y adolescentes. Se concluyó que el perfil de atractivo físico de los modelos audiovisuales (prototipos) influye en la identificación de niños y adolescentes; sin embargo, toman diferentes perspectivas de acuerdo a su edad, los niños ven los prototipos de manera global, en cambio los adolescentes evalúan los diferentes elementos faciales.

Casullo (2002) realizó una investigación sobre los criterios de elección de pareja, en donde se concluyó que los factores más relevantes son la confianza, la atracción mutua y el amor, el ser agradable y simpático, la madurez emocional. Además se encontró que los varones más jóvenes atribuyen más importancia a la atracción física que los de más edad.

\section{CONCLUSIONES}

En este estudio se ha analizado el papel que cumple la atracción en la elección de pareja, los factores que involucra y su contexto social, y se ha llegado a la conclusión de que la atracción es el punto inicial al momento de elegir una pareja, esto involucra el atractivo físico y otros factores similares como el atractivo facial, los atributos físicos, el tono de voz, etc.; sin embargo, debe considerarse también el contexto social (cultura, costumbres, valores, principios) que rodea a cada individuo, ya que eso influye en su concepto de lo que es bello.

A partir de este trabajo se puede avanzar en nuevos estudios que aporten más información en relación a la atracción y sus componentes, que permitan profundizar el tema corroborando la hipótesis planteada o formulando nuevas hipótesis. 


\section{REFERENCIAS BIBLIOGRÁFICAS}

1. Álvarez, J. (1996). La sexualidad en la pareja. México: El Manual Moderno.

2. Antaki, I. (1999). En el banquete de Platón. México: Joaquín Mortiz.

3. Attenborough, D. (2005). La vida a prueba. Madrid: RBA Editores.

4. Barriera, M., Soler, M., Jarrosay, C. \& Montoya, M. (1999). Caracterización de las relaciones de pareja en estudiantes de noveno grado de la secundaria básica Francisco Maceo Osorio. Revista Cubana de Enfermería, 15(2), 129-132. Recuperado de http://www.sld.cu/revistas/enf/vol15_2_99/ enf13299.pdf.

5. Buss, D. (2006). Evolutionary psychology. The science of the mind. Upper Saddle River, NJ: Allyn \& Bacon.

6. Casullo, M. (2002). Elección de pareja en adolescentes y adultos jóvenes. Psicodebate: Psicología, Cultura y Sociedad, (4), 39-56. Recuperado de http://www.palermo.edu/cienciassociales/publicaciones/pdf/Psico4/4\%20PSICO\%20003.pdf.

7. Díaz, P., Pandolfi, P. \& Perfetti, R. (2001). Atractivo físico. Apsique. Recuperado de http://www. aloja.cl/pdf/fisico.pdf.

8. Díaz, R. \& Sánchez, R. (2002). Psicología del amor: una visión integral de la relación de pareja. México: Porrúa.

9. Etcoff, N. (1994). Beauty and the beholder. Nature. Recuperado de ftp://tesis.bbtk.ull.es/ccssyhum/ cs77. pdf.

10. Ostrowsky, F. (2005). ¡Toc, toc! ¿Hay alguien ahí?
México: Infored.

11. Padilla, G. \& Díaz, R. (2002). La pasión, el romance y la sexualidad en las relaciones de pareja. La Psicología Social en México. México: AMEPSO.

12. Rice, F. (1997). Desarrollo humano, estudio del ciclo vital. México: Prentice-Hall.

13. Ruiz, C. (1999). Evaluación del atractivo físico de modelos audiovisuales: procesos de identificación en niños y adolescentes. Departamento de Psicología Educativa, Evolutiva y Psicobiología. Recuperado de ftp://tesis.bbtk.ull.es/ccssyhum/cs77. pdf.

14. Schopenhauer, A. (2004). El amor, las mujeres y la muerte (Trad. de A. López White). México: Coyoacán.

15. Valdez, J., González, N., Arce, J., González, S., Ison, M. \& Morelato, G. (2008). La elección de pareja real e ideal en dos culturas: México y Argentina. Un análisis por sexo. Enseñanza e Investigación en la Psicología, 13(2), 261-277. Recuperado de http://www.redalyc.org/redalyc/ pdf/292/29213205.pdf.

16. Valdez, J., González, N. \& Sánchez, Z. (2005). Elección de pareja en universitarios mexicanos. Enseñanza e Investigación en Psicología. 10(2), 355-367. Recuperado de http://redalyc.uaemex. mx/pdf/292/29210210.pdf.

17. Valdez, J., Díaz, R. \& Pérez, M. (2006). Los hombres y las mujeres en México: dos mundos distantes y complementarios. Toluca (México): Universidad Autónoma del Estado de México.

18. Winch, R. (1958). Mate selection: A theory of complementary needs. New York: Harper \& Row. 